\title{
The pressure generated during the insertion of an intramedullary device: experiments and numerical modeling
}

\author{
D. Dobrjanski ${ }^{1}$, O. Gaber ${ }^{1}$, Z. Saghir ${ }^{1}$, K. Behdinan ${ }^{2}$, P. Zalzal ${ }^{3}$, \\ J. de Beer ${ }^{4} \&$ M. Papini ${ }^{1}$ \\ ${ }^{1}$ Department of Mechanical and Industrial Engineering, \\ Ryerson University, Canada \\ ${ }^{2}$ Department of Aerospace Engineering, Ryerson University, Canada \\ ${ }^{3}$ Mount Sinai Hospital, Canada \\ ${ }^{4}$ Henderson Hospital, Canada
}

\begin{abstract}
During the orthopaedic procedures of total knee arthroplasty (TKA) and total hip arthroplasty (THA), it is often necessary to hammer an intramedullary device into the femur. This action is thought to create high pressures in the intramedullary canal, and may result in the release of fat emboli into the cardiovascular system. This work presents measured intramedullary pressures generated during hammering of an intramedullary device into a simulated femur geometry consisting of a tube of porous open-cell plastic with the same porosity and pore size as femoral cortical bone. The experimental results were found to be, on average, approximately $20 \%$ lower than those predicted by a computational fluid dynamics (CFD) model of the insertion process. Based on this, we conclude that it is reasonable to use CFD models to predict intramedullary pressures resulting from orthopaedic procedures that breach the intramedullary canal. As far as the authors know, this is the first attempt to use CFD modeling to study a problem of this type.
\end{abstract}

Keywords: fat embolism, intramedullary cavity, intramedullary nailing, intramedullary stem, computational fluid dynamics, femur. 


\section{Introduction}

In many orthopaedic procedures, it is necessary to breach the intramedullary canal of long bones, to insert prosthetic stems, intramedullary nails, and other devices. One complication of these procedures is Fat Embolism Syndrome (FES), which refers to the clinical signs and symptoms associated with the presence of fat globules within the peripheral circulation [1]. Though its etiology is not completely understood, it is generally accepted that it results from the release of the fat from the intramedullary contents into the bloodstream, due to elevated pressure within the intramedullary canal [2]. FES was first discovered by F. A. Zenker in 1862 while studying a railroad worker who had suffered a severe thoraco abdominal crush injury. Zenker found fat droplets in the pulmonary circulation of the lungs. The first clinical study was carried out by Von Bergman in 1873. Bergman described how a man suffering from a femur fracture died due to respiratory distress [3].

The incidence and mortality of FES are variable parameters from study to study. However based on a recent 10 year retrospective study, the incidence of FES was identified in $0.9 \%$ of cases with a mortality of $7 \%$. The study is based on a 10 -year review of trauma cases with a population of 3026 patients [4].

A number of investigators have found, very high pressures associated with this hammering of intramedullary devices into cadaveric specimens. During the insertion of the stem into the femoral canal pressures are seen between $70-$ $1950 \mathrm{mmHg}[5,6,7]$.

The goal of the present work was to develop experimental and numerical methods to measure and study the intramedullary pressure in the femur due to impact forces produced from this hammering action. The hope is that this model can be used to study the effect of various operative parameters (rate of insertion, hammering force, orientation of bone, etc.), device geometry (prosthetic tip shape, diameter, etc.) and bone/marrow material properties (porosity, viscosity, etc.), so that the risk of FES in orthopaedic procedures can be reduced.

\section{Materials and methods}

\subsection{Simulated bone/implant/marrow system}

In order to avoid the inter-specimen variability associated with cadaveric specimens, and to determine the feasibility of using computational fluid dynamics (CFD) to study fluid flow in bone, a simplified model system was developed. Finding a suitable cortical bone analogue proved to be a challenging task. For study of fluid flow in a model system, it was important to choose a cortical bone analogue having similar porosity, density, pore size, and modulus of elasticity to that of human cortical bone $\left(5-30 \%, 1810 \mathrm{~kg} / \mathrm{m}^{3}, 3-78\right.$ microns, and 10-20GPa, respectively). In addition, the material must be made of an open pore structure $[8,9,11]$. A large number of materials were tested; e.g.: Polyurethane foam, which resulted in $20-25 \%$ porosity, but was not open cell, and ProOsteon, BoneSource, DynaGraft, ChronOs, Vitoss, Crotoss, which were 
all too porous $(70 \%+)$, and, in addition, were too brittle. Open cell ceramics were also candidates, however, no outflow was observed through the pores to the exterior of the cylinder, and they were thus dismissed, because the flow through the pores is crucial to have a valid comparison with the computer models.

The only suitable choice found was a porous plastic, which was custom manufactured with an open cell structure having 15-30\% porosity, and 40-60 microns pore size, matching the requirements in terms of pore size and porosity quite well. Unfortunately, the plastic is not as stiff as human bone. However, for the purpose of comparison with the CFD-FIDAP model, which models fluid flow rather than mechanical behaviour and allows input of any mechanical properties, this was deemed to be acceptable. If the experimental values were found to match those predicted by the computer models using this system, there is no reason to believe that a decrease in elastic modulus would invalidate the model. A hollow cylinder of length, outer diameter, and inner diameter, $350 \mathrm{~mm}$, $32 \mathrm{~mm}$ and $16 \mathrm{~mm}$, respectively, roughly matching a large human femur, was thus constructed from this material, as used as the cortical bone analogue.

The implant chosen was a simple stainless steel cylinder with a Teflon piston end. The Teflon piston was chosen to reduce any friction between the inner walls of the cylinder if it occurs during the experiment. The piston had a diameter of $15.5 \mathrm{~mm}$, which created a $0.5 \mathrm{~mm}$ gap between the piston and the inner walls of the cylinder. A simple standard flat tip was chosen for the piston, for the sake of simplicity and to save computation time when comparing to the CFD-FIDAP model.

To reproduce the viscoelastic properties of bone marrow a petroleum jelly and paraffin mixture was used. The viscoelastic properties of this mixture at $20^{\circ} \mathrm{C}$ have been shown to be roughly equivalent to those of bone marrow fat at $36^{\circ} \mathrm{C}$ [8]. The porous plastic cylinder was filled with a mixture of constant porosity, in the range of $50 \mathrm{cP}$ to $600 \mathrm{cP}[10]$, representing the range of viscosity between the distal and proximal bone marrow in the femur. The mixture selected for the experiments was a $45 / 55$ ratio of vaseline/paraffin. The viscosity of the mixture was calculated as $82.6 \mathrm{cP}$, using a Gilmont falling ball viscometer.

\subsection{Experiments}

In order to measure the pressures resulting from hammering an intramedullary device into the cylinder, an experimental apparatus (Fig. 1) was built. A swinging pendulum with an adjustable hammer mount was constructed to achieve controlled impulse forces. Attached to the hammer mount was an impulse hammer having the capability to measure the force of the hammering, as a function of time, and a weight, which controlled the peak hammering load. The cylinder was mounted on a rail system, which allowed the cylinder to move forward, after each strike of the stem. The cylinder was tapped at four locations along the span, and a pressure transducer was inserted in those locations. The location of the taps occurred every $75 \mathrm{~mm}$ from the entrance of the cylinder (fig. 2).

To collect the experimental data, a data acquisition (DAQ) system (IOTech DaqBook 2000, A-Tech Instruments Ltd, Toronto, Canada) was coupled to a 
Pentium 4-2.6GHz computer. The DAQ system was capable of collecting 200,000 samples per second from 32 separate channels ensuring that peak pressures were not missed. The pressure values were obtained from pressure transducers (Kulite XTM190 5-Wire, Kulite Semiconductor Products Inc., Leonia, USA). The hammer impulse was obtained from an impulse hammer (Dytran 5800B4, Dytran Instruments, Inc., Chatsworth, USA) and the displacement of the implant was obtained from a draw-wire displacement sensor (MicroEpsilon WDS-500, Micro-Epsilon Messtechnik GmbH \& Co., Ortenburg, Germany). Experimental results were collected with the use of DaqView 7.13 software and analyzed with Microsoft Excel 2003.

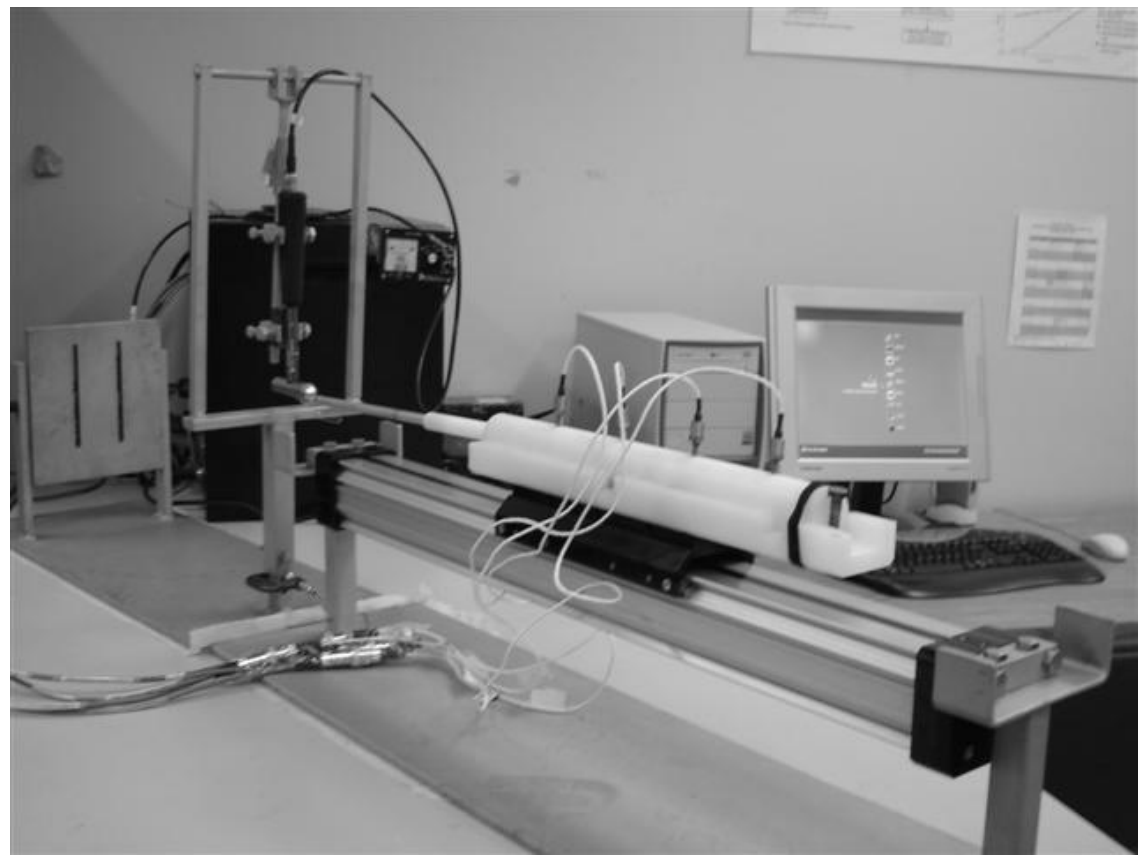

Figure 1: $\quad$ Experimental apparatus set-up.

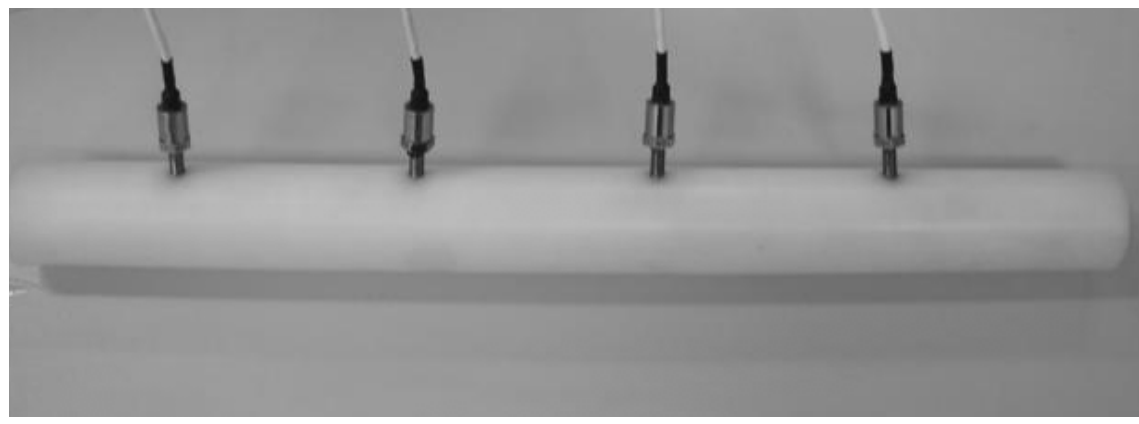

Figure 2: $\quad$ Porous plastic cylinder (pressure transducer locations). 


\subsection{Numerical model}

To duplicate the experimental data, a model of the cylinder and fluid was built in Gambit (Fluent, New Hampshire, U.S.A.) and analyzed in FIDAP (Fluent, New Hampshire, U.S.A.). Two $350 \mathrm{~mm}$ long co-axial cylinders having outer and inner diameters of $32 \mathrm{~mm}$ and $16 \mathrm{~mm}$, respectively, were used to simulate the walls of the cylinder (Fig. 3).

From the experimental data, it was observed that after the implant was struck it moved in a sinusoidal fashion, and a curve was fit to the data points (Fig. 4). The velocity of the implant was then calculated by taking the derivative of the function and used in the numerical simulation code as the implant insertion rate. The resulting velocity distribution is shown in Fig. 5. At the tip of the inner cylinder, the surface velocity was set to be equal to the insertion rate to mimic the movement of the implant as it advanced after being struck. The displacement and the velocity of the implant were defined as follows:

$$
\begin{gathered}
Z=0.00434(1-\cos (1800 t)) \\
U_{Z}=0.13635 \sin (1800 t)
\end{gathered}
$$

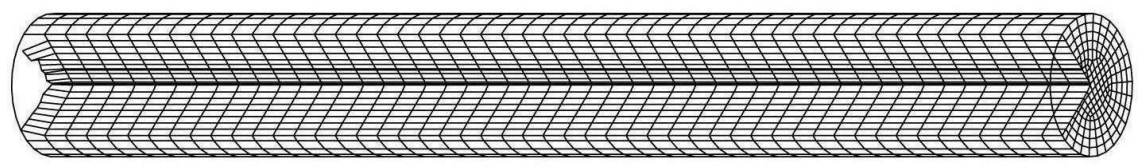

Figure 3: Numerical model of the cylinder.

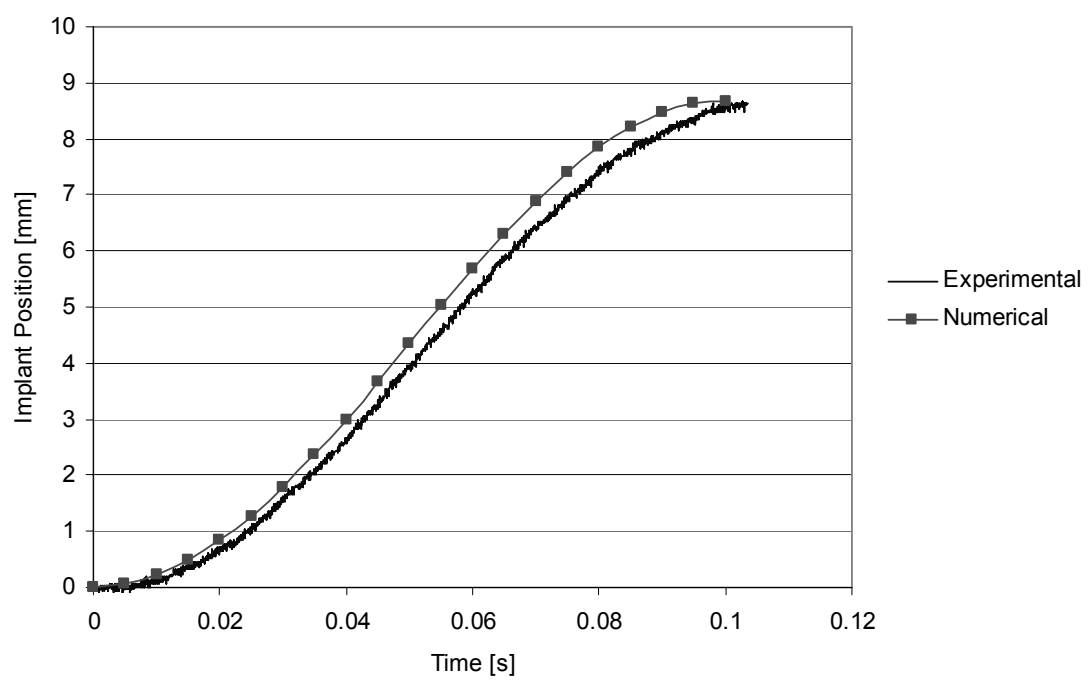

Figure 4: Experimental and numerical displacement profiles. 
The rest of the boundary conditions used in the modeling were obtained from the experimental apparatus. Velocity was specified as zero at the top and bottom of the outer cylinder and the top of the inner cylinder, so that fluid could only escape through the side walls but not through the top and bottom of the cylinders. The pressure was determined at the four locations outlined in Fig. 2, and compared to the experimental results.

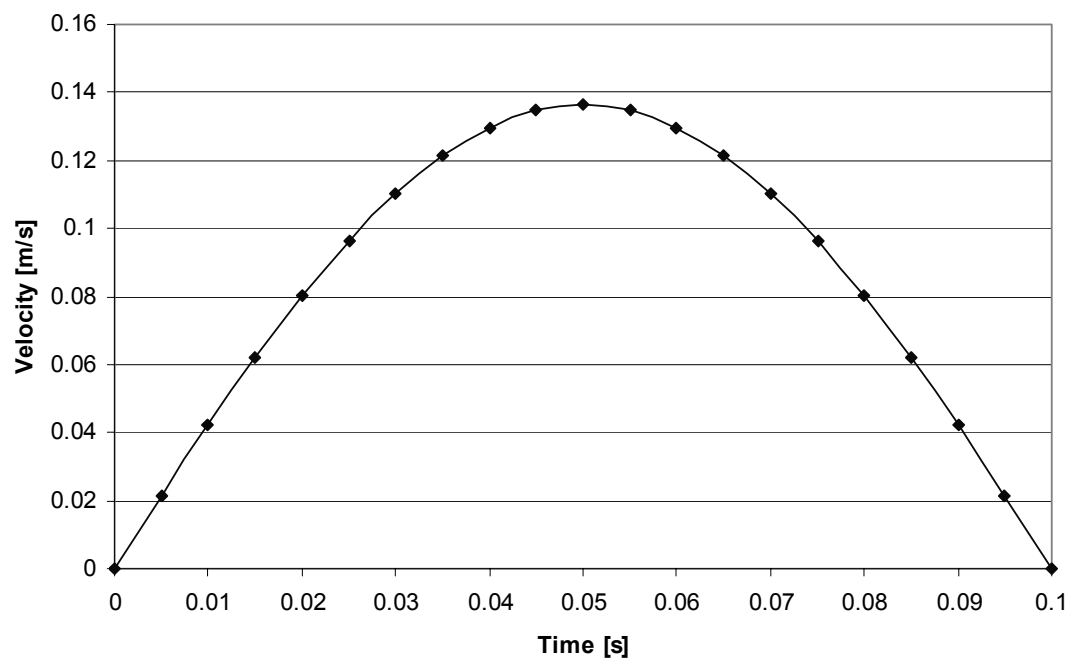

Figure 5: Numerical velocity profile.

\section{Results}

The experimental results were obtained from the transducer sensors after the hammer impacted the stem. The pressure was monitored after the impulse hammer made contact with the stem, and the displacement of the stem with respect to time was observed from the potential displacement sensor. The experiment was carried out 5 times to ensure repeatability in the results. Two examples are shown in Fig. 6 (upper and lower pressure results). In all cases, the variability between experiment runs was minimal, approximately $1.9 \%$ for pressure, and 5.6\% for displacement. The measured displacement of the stem with respect to time is shown in Fig. 4. The mean impulse of the hammer was found to be approximately $1206 \mathrm{~N}$ over a time increment of $0.25 \mathrm{~ms}$. Fig. 6 compares the experimental and numerical pressures during the insertion of the intramedullary device, with respect to the transducer number. Pressure transducer 1 was located at $75 \mathrm{~mm}$ from the front of the cylinder, whereas transducer 4 was located $300 \mathrm{~mm}$ from the front. Table 1 summarizes the differences between experimental and numerical values. The results vary from $17 \%$ to $23 \%$. 
Table 1: $\quad$ Experimental and numerical comparison of pressure results.

\begin{tabular}{|l|c|c|c|c|}
\hline & $\begin{array}{c}\text { Pressure } \\
\text { Transducer 1 } \\
{[\%]}\end{array}$ & $\begin{array}{c}\text { Pressure } \\
\text { Transducer 2 } \\
{[\%]}\end{array}$ & $\begin{array}{c}\text { Pressure } \\
\text { Transducer 3 } \\
{[\%]}\end{array}$ & $\begin{array}{c}\text { Pressure } \\
\text { Transducer 4 } \\
{[\%]}\end{array}$ \\
\hline Experiment 1 & 22.955 & 19.610 & 20.326 & 20.320 \\
\hline Experiment 2 & 21.735 & 17.634 & 18.902 & 18.725 \\
\hline
\end{tabular}

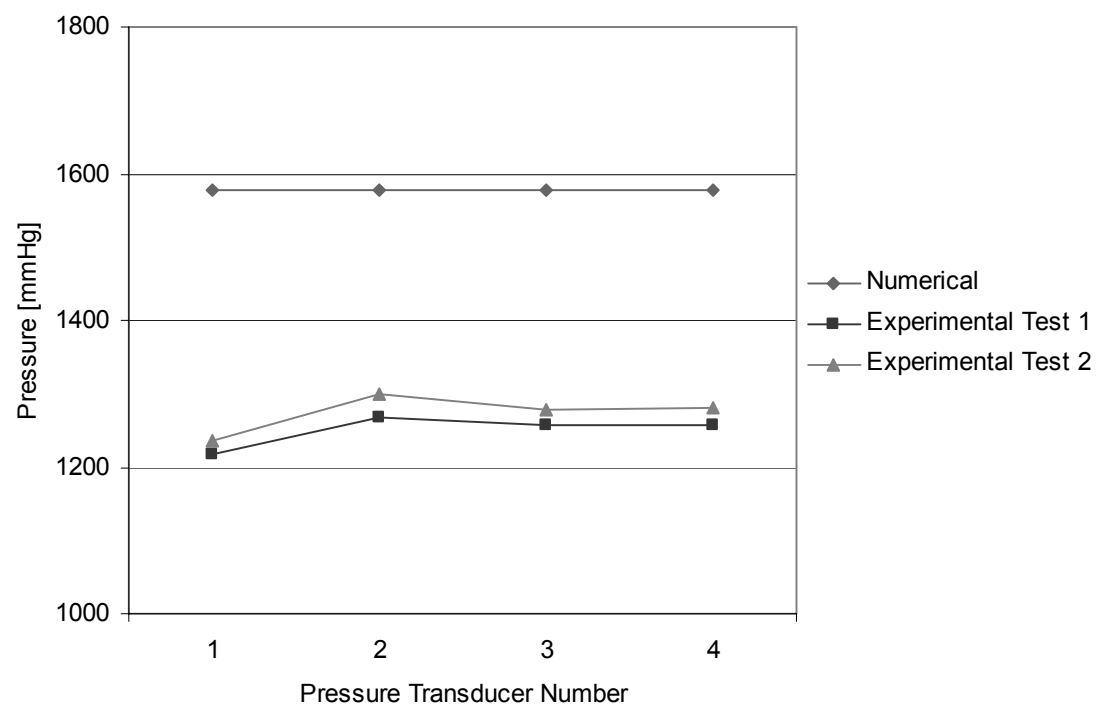

Figure 6: Experimental and numerical pressure values.

\section{Discussion}

The reasonable (approx. 20\%) correlation between the two models shows that the use of CFD models to study intramedullary pressures resulting from insertion of a device into the intramedullary canal in long bones is possible. To the knowledge of the authors, this is the first attempt to use CFD modeling to study a problem of this type.

The experimental and numerical pressure values obtained in this study fall in the range based on previous femoral nailing studies based on cadaveric specimens, which resulted in measured intramedullary pressures between $70-1950 \mathrm{mmHg}[5,6,7]$. The wide range of values in these studies are mainly due to differences in bone geometry, quality, hitting force, and the gap between the stem and the inner walls of the bone.

The present study lays the groundwork for future parametric studies to be performed using our model. The model will allow for study of the effect of parameters such as: the impulse force, tip geometry, rate of insertion, implant 
size, etc that could reduce the pressure build-up in the femoral canal. The model is currently being extended so that reaming of the intramedullary canal prior to insertion of intramedullary devices can also be simulated.

\section{Acknowledgement}

The authors would like to acknowledge the financial support of the National Science and Engineering Council (NSERC).

\section{References}

[1] Mellor A \& Soni N. Fat embolism. Anaesthesia, 56, pp. 145-154, 2001.

[2] Woo R, Minster GJ, Fitzgerald RH, Mason LD, Lucas DR \& Smith FE. Pulmonary Fat Embolism in Revision Hip Arthroplasty. Clinical Orthopaedics and Related Research, 319, pp. 41-53, 1995.

[3] Hager C.A. \& Brncick N. Fat embolism syndrome: A complication of orthopaedic trauma. Orthopaedic Nursing, pp. 41-46, 1998.

[4] Bulger E.M., Smith D.G., Maier R.V. \& Jurkovich GJ. Fat embolism syndrome. Arch. Surgery, 132, pp. 435-439, 1997.

[5] Kropfl A, Berger U, Neureiter H, Hertz H \& Schlag G. Intramedullary pressure and bone marrow fat intravasation in unreamed femoral nailing. The Journal of Trauma: Injury, Infection and Critical Care, pp. 946-954, 1997.

[6] Heim D, Chlegel U \& Perren SM. Intramedullary pressure in reamed and unreamed nailing of the femur and tibia. Injury, 24, 1994.

[7] Frolke J.M., Bakker F.C., Patka P \& Haarman H.J. Intramedullary pressure in reamed femoral nailing with two different reamer designs. European Journal of Trauma, pp. 235-240, 2001.

[8] Peltier LF. Fat embolism: A current concept. Clin Orthop, 66, pp. 241253, 1969.

[9] X, Qingwen N. Determination of cortical bone porosity and pore size distribution using a low field pulsed NMR approach. Journal of Orthopaedic Research, 21, pp. 312-319, 2003.

[10] Mousavi M, David R, Ehteshami J, Pajenda G \& Vecsei V. Pressure changes during reaming with different parameters and reamer designs. Clinical Orthopaedics and Related Research, 373, pp. 295-303, 1999.

[11] Bryant J.D., David T, Gaskell P.H., King S \& Lond G. Rheology of bovine bone marrow. Proc Instn Mech Engrs, 203, pp. 71-75, 1989. 